\title{
Differentialgleichungen unendlich hoher Ordnung mit konstanten Koeffizienten
}

\section{Doctoral Thesis}

Author(s):

Muggli, Hermann

Publication date:

1938

Permanent link:

https://doi.org/10.3929/ethz-a-000091183

Rights / license:

In Copyright - Non-Commercial Use Permitted 


\title{
Differentialgleichungen unendlich hoher Ordnung mit konstanten Koeffizienten
}

\author{
Von der \\ Eidgenössischen Technischen Hochschule in Zürich \\ zur Erlangung der Würde eines Doktors der Mathematik
} genehmigte

\section{Promotionsarbeit}

vorgelegt von

HERMANN MUGGLI

aus Bäretswil und Rüschlikon (Zürich)

Referent: Herr Prof. Dr. G. Pólya

Korreferent: Herr Prof. Dr. W. Saxer

$\begin{array}{llll}1 & 9 & 3 & 8\end{array}$

ORELLFUSSLIVERLAG, Z UR I CH 
Separatabdruck aus Commentarii Mathematici Helvetici vol. 11, fasc. 2 


\section{Einleitung}

1. Die vorliegende Arbeit behandelt lineare Differentialgleichungen unendlich hoher Ordnung mit konstanten Koeffizienten, d. h. Gleichungen von der Form

$$
l_{0} F(z)+l_{1} F^{\prime}(z)+l_{2} F^{\prime \prime}(z)+\cdots+l_{\nu} F^{(p)}(z)+\cdots=G(z) .
$$

Die Koeffizienten $l_{0}, l_{1}, l_{2}, \ldots, l_{\nu}, \ldots$ sind gegebene, im allgemeinen komplexe Zahlen. Wir beschränken uns auf den Fall, in welchem $F(z)$ und $G(z)$ analytische Funktionen der komplexen Variablen $z$ sind.

Die linke Seite von (1) wollen wir als das Resultat einer auf $F(z)$ angewandten linearen Funktionaloperation auffassen, die wir mit $\mathfrak{L}$ bezeichnen; wir setzen also

$$
l_{0} F(z)+l_{1} F^{\prime}(z)+l_{2} F^{\prime \prime}(z)+\cdots+l_{\nu} F^{(\nu)}(z)+\cdots=\mathfrak{L} F(z) .
$$

Die Funktionaloperation $\mathfrak{L}$ ist durch die Angabe der Zahlenfolge $l_{0}, l_{1}, l_{2}, \ldots, l_{\nu}, \ldots$ bestimmt, oder, was damit gleichbedeutend ist, durch die Angabe der Potenzreihe

$$
l_{0}+l_{1} z+l_{2} z^{2}+\cdots+l_{\nu} z^{\nu}+\cdots=L(z),
$$

welche wir die erzeugende Funktion der Funktionaloperation $\mathfrak{L}$ nennen wollen.

Man sagt, die Funktionaloperation $\mathfrak{Q}$ sei auf die Funktion $F^{\prime}(z)$ anwendbar, wenn die Reihe links in (2) in jedem regulären Punkt $z$ von $F(z)$ konvergiert.

2. Soll die Funktionaloperation $\mathfrak{L}$ auf alle Funktionen einer gewissen Klasse anwendbar sein, so muß die Koeffizientenfolge $l_{0}, l_{1}, l_{2}, \ldots$ eine dieser Klasse entsprechende Bedingung erfüllen. In dieser Arbeit werden vier verschiedene Funktionenklassen behandelt, nämlich die folgenden:
A) beliebige reguläre Funktionen;
B) beliebige ganze Funktionen;
C) ganze Funktionen endlicher Ordnung;
D) ganze Funktionen vom Exponentialtypus (d.h. höchstens vom Mitteltypus der Ordnung 1).


Die Bedingung für die Anwendbarkeit der Operation $\mathfrak{L}$ kann man in allen vier Fällen vollständig aufstellen und in doppelter Weise ausdrücken, sowohl als eine Bedingung für die Koeffizientenfolge $l_{0}, l_{1}, l_{2}, \ldots$ wie auch als Bedingung für die erzeugende Funktion $L(z)$. Es gilt nämlich, wie wir beweisen werden, der folgende, die vier Fälle zusammenfassende

Satz I. A. Die Operation $\mathfrak{I}$ ist dann und nur dann auf alle regulären Funktionen $F(z)$ anwendbar, wenn

$$
\lim _{\nu \rightarrow \infty}\left|\nu ! l_{\nu}\right|^{\frac{1}{\nu}}=0
$$

gilt, oder, was damit gleichbedeutend ist, wenn $L(z)$ eine ganze Funktion ist, deren Anwachsen den Minimaltypus der Ordnung 1 nicht übersteigt.

B. Die Operation $\mathfrak{I}$ ist dann und nur dann auf alle ganzen Funktionen $F(z)$ anwendbar, wenn

$$
\left|v ! l_{\nu}\right|^{\frac{1}{v}} \quad \text { beschränkt }
$$

bleibt, oder, was damit gleichbedeutend ist, wenn $L(z)$ eine ganze Funktion vom Exponentialtypus ist.

C. Die Operation $\mathfrak{Q}$ ist dann und nur dann auf alle ganzen Funktionen $F(z)$, deren Ordnung @ nicht übersteigt, wobei @ $\geqq 1$, anwendbar, wenn

$$
\varlimsup_{\nu \rightarrow \infty}\left|l_{v}\right|^{\frac{1}{\nu \log \nu}}<e^{\frac{1}{\varrho}-1}
$$

ist. Im Fall $\varrho>1$ besagt dies, daß die Ordnung von $L(z)$ kleiner als $\varrho^{\prime}$ ist, wobei

$$
\frac{1}{\varrho}+\frac{1}{\varrho^{\prime}}=1 ;
$$

falls $\varrho=1$, so besagt die Bedingung (c), daß $L(z)$ von endlicher Ordnung ist.

D. Die Operation $\mathfrak{Q}$ ist dann und nur dann auf alle ganzen Funktionen, deren Anwachsen den Typus $t$ der Ordnung 1 nicht übersteigt, anwendbar, wenn

$$
\varlimsup_{\nu \rightarrow \infty}\left|l_{\nu}\right|^{\frac{1}{\nu}}<\frac{1}{t}
$$

ist ; im Fall $t=0$ ist diese Bedingung so zu verstehen, daß $\left|l_{v}\right|^{1 / \nu}$ beschränkt bleibt. Die Bedingung (d) besagt, daß $L(z)$ im Kreis $|z| \leqq t$ regulär ist. (Sie besagt insbesondere im Fall $t=0$, daß $L(z)$ im Punkt $z=0$ regulär ist.)

Wir werden ferner beweisen, daß die Operation $\mathscr{L}$ in allen vier Fällen 
eine Funktion $F(z)$ der betreffenden Klasse in eine Funktion $\mathcal{L} F(z)$ derselben Klasse verwandelt. Genauer gesagt, besteht der

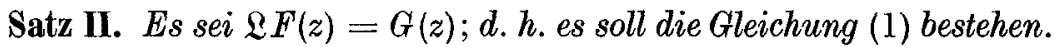

A. Wenn (a) gilt, so ist $G(z)$ auf jeder Stelle $z$, auf welcher $F(z)$ regulär ist, ebenfalls regulär.

B. Wenn (b) gilt und $F(z)$ eine ganze Funktion ist, so ist $G(z)$ ebenfalls ganz (und zwar von nicht höherer Ordnung und nicht höherem Typus als $F(z))$.

C. Wenn (c) gill und $F(z)$ eine ganze Funktion von der Ordnung $\varrho$ ist, wobei $\varrho \geqq 1$, so ist $G(z)$ eine ganze F unktion und höchstens von der Ordnung $\varrho$.

D. Wenn (d) gilt und $F(z)$ eine ganze Funktion ist, so beschaffen, daß ihr Anwachsen den Typus $t$ der Ordnung 1 nicht übersteigt, so ist $G(z)$ eine ganze Funktion von der selben Beschaffenheit.

Zusätze zu Satz II. D 1. Unter den Voraussetzungen des Falles D kann man weiter behaupten, daß das Indikatordiagramm von $G(z)$ ein (echter oder unechter) Teilbereich des Indikatordiagrammes von $F(z)$ ist.

D 2. Wenn (d) gilt und $F(z)$ eine ganze Funktion von der Ordnung @ ist, wobei $\varrho<1$, so ist $G(z)$ eine ganze Funktion und höchstens von der Ordnung $\varrho$.

Die Sätze I und II werden, insofern sie sich mit den Fällen A, B, C befassen, schon im ersten Kapitel bewiesen. Die Beweise im Fall D müssen auf das III. Kapitel verschoben werden. Vorher sind nämlich noch die wichtigsten Punkte aus der Theorie der ganzen Funktionen vom Exponentialtypus und ihrer Indikatordiagramme (vgl. [9])ㄱ) zu besprechen $\left.(\mathrm{Nr} .15)^{2}\right)$.

1) Die fetten Ziffern in eckigen Klammern beziehen sich auf das Literaturverzeichnis auf S. 156.

2) Hier sei die Bemerkung eingeschaltet, daß in den Fällen $A$ und $B$ die Operation $\mathfrak{L}$ ,,beschränkt" und ,stetig" ist; vgl. [9, S. 600, Satz VI]. In den Fällen $C$ und $D$ besteht kein derartiger Satz. Dies zeigt das folgende Beispiel, das man zu beiden Fällen $C$ und $D$ rechnen kann: Die Operation $\mathfrak{E}$ werde durch

erzeugt und auf die Funktionenfolge

$$
L(z)=e^{z^{2}}
$$

$$
F_{n}(z)=\frac{z^{2 n}}{n !} \quad(n=1,2,3, \ldots)
$$

angewandt. Das ergibt

$$
\mathfrak{L} F_{n}(z)=\frac{z^{2 n}}{n !}+\cdots+\frac{(2 n) !}{(n !)^{2}} \text {. }
$$

Obwohl $F_{n}(z)$ in jedem festen endlichen Bereich mit wachsendem $n$ gleichmäßig gegen Null konvergiert, ist $\mathfrak{L} F_{n}(z)$ im Nullpunkt der $z$-Ebene ersichtlich unbeschränkt. 
3. Man kann in der Gleichung (1) die Funktion $G(z)$ und die Koeffizientenfolge $l_{0}, l_{1}, l_{2}, \ldots$ als gegeben betrachten und eine Funktion $F(z)$ suchen, welche die Gleichung befriedigt. Wenn eine solche Funktion $F(z)$ existiert, so kann sie keinen ausgedehnteren Regularitätsbereich haben (Fall A), bzw. nicht von langsamerem Anwachsen sein (Fälle B, C, D) als die Funktion $G(z)$; dies besagt gerade der Satz II. Existiert aber eine Funktion $F(z)$, welche (1) genügt, im selben Gebiet wie $G(z)$ regulär ist und auch kein schnelleres Anwachsen als $G(z)$ zeigt? Diese Frage wird zum guten Teil durch den folgenden Existenzsatz, dessen Beweis den Hauptinhalt dieser Arbeit ausmacht, beantwortet:

Satz III. A. Wenn (a) gilt und $G(z)$ in einer gegebenen offenen Kreisfläche $\Omega$ der $z$-Ebene regulär ist, so besitzt die Gleichung (1) eine Partikularlösung, welche in $\Omega$ ebenfalls regulär ist.

B. Wenn (b) gilt und $G(z)$ eine ganze Funktion ist, so besitzt die Gleichung (1) eine Partikularlösung, welche ebenfalls eine ganze Funktion ist.

C. Wenn (c) gilt und $G(z)$ eine ganze Funktion von der Ordnung $\varrho$ ist, wobei $\varrho \geqq 1$, so besitzt die Gleichung (1) eine Partikularlösung, welche ganz und ebenfalls von der Ordnung $\varrho$ ist.

D. Wenn (d) gilt und $G(z)$ eine ganze Funktion ist, höchstens vom Typus $t$ der Ordnung 1, so besitzt die Gleichung (1) eine Partikularlösung, deren Indikatordiagramm mit dem Indikatordiagramm von $G(z)$ zusammenfällt.

Zusätze zu Satz III. A. B. Unter den Voraussetzungen des Falles A wie auch unter denen des Falles $B$ gibt es eine unendlich vielparametrige lineare Schar von Partikularlösungen der genannten Art, abgesehen vom einzigen Ausnahmefall, in welchem die Gleichung (1) mit einer linearen Differentialgleichung endlicher Ordnung für $F(z+c)$, mit konstantem $c$, gleichwertig iet.

D 1. Es seien die Voraussetzungen des Falles $D$ erfüllt. Ferner sei ein abgeschlossener konvexer Bereich $\mathfrak{B}$ gegeben, welcher im Kreis $|z| \leqq t$ enthalten ist und das Indikatordiagramm von $G(z)$ enthält. Eine ganze Funktion $F(z)$ vom Exponentialtypus, deren Indikatordiagramm im vorgegebenen Bereich $\mathfrak{B}$ enthailten ist, ist dann und nur dann eine Lösung der Gleichung (1), wenn sie folgende Gestalt hat:

$$
F(z)=F_{0}(z)+P_{m_{1}}(z) e^{\lambda_{1} z}+\cdots+P_{m_{l}}(z) e^{\lambda_{l} z} .
$$

Hiebei bedeutet $F_{0}(z)$ eine fest gewählte Partikularlösung von (1), deren Indikatordiagramm mit dem von $G(z)$ zusammenfällt $; \lambda_{1}, \lambda_{2}, \ldots, \lambda_{l}$ sind 
diejenigen verschiedenen Nullstellen von $L(z)$, welche im Bereich $\overline{\mathfrak{B}}$, dem Spiegelbild von $\mathfrak{B}$ in bezug auf die reelle Achse, liegen; die $m_{1}, m_{2}, \ldots, m_{l}$ bezeichnen die Vielfachheiten von $\lambda_{1}, \lambda_{2}, \ldots, \lambda_{l}$ und $P_{m_{1}}(z), P_{m_{2}}(z), \ldots$, $P_{m_{l}}(z)$ sind Polynome vom Grad $m_{1}-1, m_{2}-1, \ldots, m_{l}-1$ mit willkürlichen Koeffizienten. (Die Anzahl der Parameter der linearen Schar (4) ist gleich der Anzahl der mit Mehrfachheit gezählten Nullstellen von $L(z)$ in $\overline{\mathfrak{B}}$.)

D 2. Wenn (d) gilt und $G(z)$ eine ganze Funktion von der Ordnung $\varrho$ ist, wobei $\varrho<1$, so hat die Gleichung (1) eine Partikularlösung $F_{0}(z)$ von der

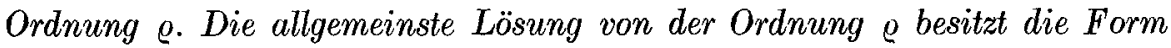
$F_{0}(z)+P_{m}(z)$. Dabei bedeutet $P_{m}(z)$ ein Polynom vom Grad $m-1$ mit willkürlichen Koeffizienten und $m$ die Vielfachheit, mit der $L(z)$ im Nullpunkt verschwindet. (Wenn $L(0) \neq 0$ ist, so ist $F_{0}(z)$ die einzige Lösung von der Ordnung $\varrho$.)

4. Die Literatur über lineare Differentialoperatoren und -Gleichungen unendlich hoher Ordnung ist sehr ausgedehnt und kann hier nicht ausführlich besprochen werden. Ein Überblick über das Gebiet findet sich in [2], ein ausführliches Literaturverzeichnis in [3]. Es soll daher nur kurz erläutert werden, inwiefern diese Arbeit Neues enthält.

Daß die in Satz I ausgesprochenen Bedingungen hinreichend sind, wurde schon von verschiedenen Autoren erkannt; daß die selben Bedingungen auch notwendig sind, scheint weniger bekannt zu sein. Von Satz II dürften die Aussage im Fall C sowie die Zusätze D 1 und D 2 neu sein.

Die erste allgemeine Methode zur Lösung von Differentialgleichungen unendlich hoher Ordnung mit konstanten Koeffizienten gab Schürer in [10]. Die Methode ist nicht an analytische Funktionen gebunden; sie beruht aber auf gewissen Voraussetzungen, die auf analytische Funktionen angewandt denen des Falles $\mathbf{D}$ entsprechen. Die Arbeiten von Hilb, Perron und Helge von Koch über Differentialgleichungen unendlich hoher Ordnung mit Polynomen beschränkten Grades als Koeffizienten betreffen einen allgemeineren Fall; wenn man sie zu dem (wesentlich einfachern) Fall der konstanten Koeffizienten spezialisiert, kommt man wieder zu den Bedingungen des Falles D. Die Aussagen des Satzes III im Fall D und des Zusatzes D 1 sind immerhin insofern neu, als sie sich auf das Indikatordiagramm beziehen; ebenso ist Zusatz $\mathrm{D} 2$ neu. Als Grundlage für die Beweise diente mir die Arbeit [9] von Prof. Pólya.

Die Methode, die wir in den Fällen $\mathrm{A}$ bis $\mathrm{C}$ anwenden werden, ist dieselbe, die Hurwitz in [5] zur Lösung der Differenzengleichung $F(z+1)-$ 
$F(z)=G(z)$ in den Fällen B und D benützte. (Für diese Differenzengleichung hat übrigens Whittaker in [12] auch den Fall $\mathrm{C}$ behandelt; ferner hat er in [13] den betreffenden Spezialfall des Zusatzes D 2 zu Satz III hergeleitet.) Der Hurwitzsche Ansatz wurde schon 1936 von Carmichael zur Lösung von allgemeinen Differentialgleichungen unendlicher Ordnung benutzt; vgl. [1]. Doch darf ich erwähnen, daß mich Herr Prof. Pólya schon im Frühjahr 1935 auf diese Methode aufmerksam machte. Carmichael ist Satz III B sehr nahe gekommen, dagegen ist der Satz III in den Fällen A und C ganz neu.

\section{I T E R A T U R}

1. Carmichael, R.D., On Non-homogeneous Linear Differential Equations of Infinit Order with Constant Coefficients. American Journal of Math. Bd. 58 (1936), S. $473-486$.

2. Carmichael, R.D., Linear Differential Equations of Infinit Order. Bull. American Math. Soc. Bd. 42 (1936), S. 193-218.

3. Davis, H.T., The Theory of Linear Operators.

4. Hilb, E., Lineare Differentialgleichungen unendlich hoher Ordnung mit ganzen rationalen Koeffizienten. Math. Annalen Bd. 82 (1920-21), S. 1-39 und Bd. 84 (1921), S. 16-30 und S. 43-52.

5. Hurwitz, A., Sur l'intégrale finie d'une fonction entière. Acta Math. Bd. 20 (1897), S. 285-312. Gesammelte Abhandlungen Bd. 1, S. 436-459.

6. Kitagawa, T., On the Theory of Linear Translatable Functional Equations and Cauchy Series. Japanese Journal of Mathem. Bd.XIII, Nr. 3 (1937), S. $223-332$.

7. Helge von Koch, Sur les équations différentielles linéaires d'ordre infini. Arkiv för Math. Astron. och Fysik, Bd. 16 (1922).

8. Perron, $O$., Lineare Differentialgleichungen unendlich hoher Ordnung mit ganzen rationalen Koeffizienten. Math. Annalen, Bd. 84 (1921), S. $31-42$.

9. Pólya, $G$., Untersuchungen über Lücken und Singularitüten von Potenzreihen. Math. Zeitschrift, Bd. 29 (1929), S. 549-640.

10. Schürer, $F$., Eine gemeinsame Methode zur Behandlung gewisser Funktionalgleichungsprobleme. Berichte der sächsischen Gesellschaft der Wissenschaften, Bd. 70 (1918), S. 185-240.

11. Valiron, $G$., Integral functions.

12. Whittaker, J. M., On the Asymptotic Period of an Integral Function. Proc. of the Edinburgh Math. Soc., Bd. 3 (2. ser.) (1934), S. 241.

13. Whittaker, J.M., Note on the Sum of an Integral Function. Proc. Edinburgh Math. Soc., Bd. 4 (2. ser.), S. 77. 
I.

\section{Klassifikation und Eigensehaften der Funktionaloperation $\mathfrak{\&}$}

5. Wir wollen zunächst beweisen, daß die im Satz I genannten Bedingungen für die Anwendbarkeit der Operation $\mathfrak{L}$ auf die betreffenden Funktionenklassen notwendig sind. Jeder der vier Fälle A, B, C, D gibt zu zwei Bedingungen Anlaß, von denen die eine die Koeffizientenfolge $l_{0}, l_{1}, l_{2}, \ldots$, die andere die erzeugende Potenzreihe $L(z)$ betrifft. Daß diese beiden Bedingungen in allen vier Fällen einander äquivalent sind, dürfen wir aus der Theorie der ganzen Funktionen als bekannt voraussetzen. Somit haben wir uns nur mit den Bedingungen zu befassen, welche die Koeffizientenfolge betreffen, d. h. mit den Bedingungen (a), (b), (c), (d).

Wir verfahren indirekt und nehmen an, daß in einem der Fälle A bis D die Folge der $l_{v}$ die entsprechende Bedingung nicht erfüllt. Das würde heißen, es gäbe eine Teilfolge

$$
\begin{aligned}
& l_{\nu_{1}}, \quad l_{\nu_{2}}, \quad l_{\nu_{3}}, \ldots \\
& l_{\nu_{k}} \neq 0 \text { für } k=1,2,3, \ldots
\end{aligned}
$$

mit folgender Eigenschaft:

im Fall A

$$
\left|v_{k} ! l_{\nu_{k}}\right|^{\frac{1}{\nu_{k}}}>\varepsilon>0 ; k=1,2,3, \ldots ;
$$

im Fall B

$$
\lim _{k \rightarrow \infty}\left|v_{k} ! l_{\nu_{k}}\right|^{\frac{1}{\nu_{k}}}=\infty \text {; }
$$

im Fall C

$$
\lim _{k \rightarrow \infty}\left|l_{\nu_{k}}\right|^{\frac{1}{\nu_{k} \log \nu_{k}}} \geqq e^{\frac{1}{\varrho}-1} ;
$$

im Fall D

$$
\lim _{k \rightarrow \infty}\left|l_{\nu_{k}}\right|^{\frac{1}{v_{k}}}\left\{\begin{array}{l}
\geqq \frac{1}{t} \text { für } t>0, \\
=\infty \text { für } t=0 .
\end{array}\right.
$$

Unter diesen Voraussetzungen stellt die Potenzreihe

$$
F(z)=c_{0}+c_{1} z+c_{2} z^{2}+\cdots,
$$

deren Koeffizienten $c_{\nu}$ durch die Gleichungen

$$
\begin{aligned}
\left|c_{\nu_{k}}\right| & =\frac{1}{\left|l_{\nu_{k}}\right| \nu_{k} !}, \\
c_{\nu_{k}} l_{\nu_{k}} & =\left|c_{\nu_{k}} l_{\nu_{k}}\right|, \\
c_{\nu} & =0 \text { für } \nu \neq v_{k}
\end{aligned}
$$


bestimmt sind, in allen vier Fällen eine Funktion dar, auf welche nach Voraussetzung die Operation $\mathfrak{L}$ anwendbar sein sollte. Es ist nämlich im Fall A

$$
\varlimsup_{\nu \rightarrow \infty}\left|c_{\nu}\right|^{\frac{1}{v}}=\varlimsup_{k \rightarrow \infty} \frac{1}{\left|l_{\nu_{k}} v_{k}\right|^{\frac{1}{v_{k}}}} \leqq \frac{1}{\varepsilon}
$$

d. h. $F(z)$ ist im Nullpunkt regulär. Im Fall B wird

$$
\varlimsup_{\nu \rightarrow \infty}\left|c_{\nu}\right|^{\frac{1}{v}}=\lim _{k \rightarrow \infty} \frac{1}{\left|l_{\nu k} \nu_{k} !\right|^{\frac{1}{\nu_{k}}}}=0
$$

$F(z)$ ist somit eine ganze Funktion. Im Fall $\mathrm{C}$ ist ${ }^{3}$ )

$\varlimsup_{\nu \rightarrow \infty}\left|c_{\nu}\right|^{\frac{1}{\nu \log \nu}}=\lim _{k \rightarrow \infty}\left|l_{\nu_{k}}\right|^{\frac{-1}{\nu_{k} \log \nu_{k}}} \cdot \lim _{k \rightarrow \infty}\left(\nu_{k} !\right)^{\frac{-1}{\nu_{k} \log \nu_{k}}} \leqq e^{1-\frac{1}{\varrho}} \lim _{\nu \rightarrow \infty}\left(\frac{e}{\frac{e}{\nu \log \nu}}=e^{-\frac{1}{\varrho}}\right.$

d. h. $F(z)$ ist höchstens von der Ordnung $\varrho$. Im Fall D wird

$$
\varlimsup_{\nu \rightarrow \infty}\left|c_{\nu} \nu !\right|^{\frac{1}{v}}=\lim _{k \rightarrow \infty} \frac{1}{\left|l_{\nu_{k}}\right|^{\frac{1}{\nu_{k}}}} \leqq t
$$

$F(z)$ ist also höchstens vom Typus $t$ der Ordnung 1.

Infolgedessen müßte die linke Seite von (2) für $z=0, \mathrm{~d}$. h. die Reihe

$$
\begin{aligned}
{[\mathfrak{S F}(z)]_{z=0} } & =\sum_{\nu=0}^{\infty} l_{\nu} \nu ! c_{\nu}=\sum_{k=0}^{\infty}\left|c_{\nu_{k}} l_{\nu_{k}}\right| \nu_{k} ! \\
& =1+1+1+\cdots
\end{aligned}
$$

konvergieren. Das ist aber nicht der Fall. Dies zeigt, daß die Bedingungen (a), (b), (c), (d) des Satzes I notwendig sind.

6. Wir wollen nun beweisen, daß die im Satz I formulierten Bedingungen in den Fällen A, B, C auch hinreichend sind. Eine Ausgestaltung unserer Überlegungen wird uns sofort zu den entsprechenden Behauptungen des Satzes II führen.

A. Es sei (a) erfüllt und $F(z)$ im Punkt $z$ regulär. Dann gibt es einen abgeschlossenen Kreis vom Mittelpunkt $z$, in welchem $F(z)$ regulär ist;

3) Ich benutze im folgenden häufig die für $n \geqq 1$ gültige doppelte Ungleichung

$$
n^{n} e^{-n} \leqq n ! \leqq(n+2) n^{n} e^{-n} \text {. }
$$


bezeichnen wir seinen Radius mit $2 \delta$ und das Maximum von $|F(z)|$ in diesem Kreis mit $M$. Nach Cauchy gilt

Für genügend große $v$ ist wegen (a)

$$
\left|F^{(\nu)}(z)\right| \leqq \frac{\nu ! M}{(2 \delta)^{\nu}}
$$

$$
\nu !\left|l_{\nu}\right|<\delta^{\nu}
$$

also

$$
\left|l_{\nu} F^{(\nu)}(z)\right|<\left|l_{\nu}\right| \frac{\nu ! M}{(2 \delta)^{\nu}}<\frac{M}{2^{\nu}} .
$$

Rechts steht das allgemeine Glied einer konvergenten Reihe. Damit haben wir eine konvergente Majorante für die Reihe (2) erhalten und bewiesen, was in Satz I über den Fall A behauptet wurde.

Wenn nun ein abgeschlossener Bereich $\mathfrak{B}$ gegeben ist, worin $F(z)$ regulär ist, so bezeichne man die Distanz dieses Bereiches $\mathfrak{B}$ vom nächsten singulären Punkt von $F(z)$ mit $3 \delta$. Man nenne ferner $M$ das Maximum von $\left|F^{\prime}(z)\right|$ in demjenigen Bereich, dessen Punkte von $\mathfrak{B}$ höchstens die Distanz $2 \delta$ haben. Die vorangehende Rechnung erweist die gleichmäßige Konvergenz der Reihe (1) in $\mathfrak{B}$, also die Regularität von $G(z)$ in allen innern Punkten von $\mathfrak{B}$, d. h. die den Fall A betreffende Aussage des Satzes II.

B. Es sei (b) erfüllt und $F(z)$ eine ganze Funktion. Dann gibt es eine Zahl $\Delta$ mit der Eigenschaft, daß für alle $\nu$

$$
\nu !\left|l_{\nu}\right|<\Delta^{\nu}
$$

ist. Mit $M(r)$ werde wie üblich das Maximum von $|F(z)|$ auf dem Kreis $|z|=r$ bezeichnet. Wenn $|z| \leqq r$ ist, gelten folgende Ungleichungen:

$$
\begin{gathered}
\left|F^{(\nu)}(z)\right| \leqq \frac{\nu ! M(r+2 \Delta)}{(2 \Delta)^{\nu}}, \\
\left|l_{\nu} F^{(\nu)}(z)\right| \leqq \frac{v !\left|l_{\nu}\right| M(r+2 \Delta)}{(2 \Delta)^{\nu}}<\frac{M(r+2 \Delta)}{2^{v}} .
\end{gathered}
$$

Die Reihe (1) besitzt daher zu jedem gegebenen $r$ eine im Kreis $|z| \leqq r$ konvergente numerische Majorante. Damit sind die Sätze I und II im Fall B bewiesen.

Aus der vorangehenden Rechnung folgt auch, daß die Ordnung von $\mathfrak{Q} F(z)$ die von $F(z)$ nicht übersteigt und daß bei gegebener Ordnung das Entsprechende vom Typus gilt. Wenn nämlich die bei der Definition der Ordnung bzw. des Typus geforderten asymptotischen Ungleichungen 
von $M(r)$ erfüllt werden, so werden sie, $\Delta$ als konstant vorausgesetzt, auch von $M(r+2 \Delta)$ erfüllt.

C. Es sei die Bedingung (c) erfüllt. Wenn daher die positive aber genügend kleine Zahl $\delta$ gegeben ist, so besteht für $v \geqq \nu_{1}$ die Ungleichung

oder anders geschrieben

$$
\left|l_{\nu}\right|^{\frac{1}{\nu \log \nu}}<e^{\frac{1}{0+2 \delta}-1}
$$

$$
\left|l_{\nu}\right|<\nu^{\frac{\nu}{e+2 \delta}-\nu} .
$$

Wir führen noch die $\operatorname{Zahl} \Lambda=\operatorname{Max}\left|l_{\nu}\right|$ ein, so daß also für $y=0,1,2, \ldots$ gilt.

$$
\left|l_{v}\right| \leqq \Lambda
$$

Es sei $F(z)$ eine ganze Funktion der Ordnung $\varrho$. Es gibt daher eine Zahl $r_{1}$, so daß für $|z| \geqq r_{1}$ die Ungleichung

$$
|F(z)|<\left.e^{|z|}\right|^{e+\delta}
$$

richtig ist. Ist $2 r>r_{1}$ und $|z| \leqq r$, so gilt

$$
\left|F^{(\nu)}(z)\right|<\frac{\nu ! e^{(2 r)^{\rho+\delta}}}{r^{\nu}} .
$$

Wir setzen

$$
(2 r)^{a+\delta}=v
$$

und erhalten

$$
\left|F^{(v)}(z)\right|<v ! e^{\nu} 2^{v} v^{\frac{-\nu}{e+\delta}} \text { für } \quad v>r_{1}{ }^{e+\delta}=v_{2},|2 z|^{e+\delta} \leqq \nu .
$$

Die Reihe (2) wird jetzt in folgender Weise in zwei Teile zerlegt:

$$
\mathfrak{L} F(z)=\sum_{\nu<|2 z|^{\varrho+\delta}} l_{\nu} F^{(\nu)}(z)+\sum_{\nu \geqslant|2 z|^{\varrho+\delta}} l_{\nu} F^{(\nu)}(z) .
$$

Auf die erste Summe sind (1.2) und (1.3), auf die zweite (1.1) und (1.4) anzuwenden. Das ergibt für $|2 z|>\operatorname{Max}\left(1, r_{1}\right)$ die Abschätzungen

$$
\begin{aligned}
& |\mathcal{E} F(z)| \leqq \sum_{\nu<|2 z|^{\varrho}+\delta} \Lambda \frac{\nu ! e^{|2 z|^{\varrho+\delta}}}{|z|^{\nu}}+\sum_{\nu \geqq|2 z| \varrho+\delta} \nu^{\frac{\nu}{\varrho+2 \delta}} v !(2 e)^{\nu} v^{\frac{-\nu}{\varrho+\delta}}
\end{aligned}
$$

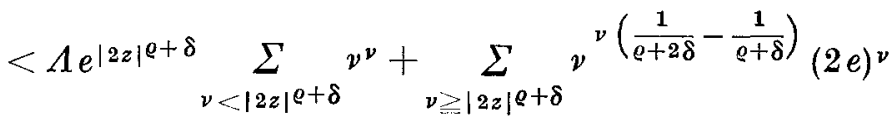

$$
\begin{aligned}
& <A e^{|2 z| \varrho+\delta}\left(|2 z|^{\varrho+\delta}+1\right)\left(|2 z|^{\varrho+\delta}\right)^{|2 z| \varrho+\delta} \\
& +\sum_{\nu=1}^{\infty} e^{-\left(\frac{1}{\varrho+\delta}-\frac{1}{\varrho+2 \delta}\right) \nu \log \nu+\nu(\log 2+1)}
\end{aligned}
$$


Daraus folgt, daß die Reihe (2) in der ganzen endlichen $z$-Ebene konvergiert, und daß ihre Summe für genügend große $|z|$ die Ungleichung

$$
|\mathfrak{L F}(z)|<e^{|z|^{\varrho+2 \delta}}
$$

befriedigt. Damit ist im Fall C Satz I und, weil $\delta$ beliebig klein sein darf, auch Satz II bewiesen.

7. Um die Zusätze zu Satz III bewreisen zu können, müssen wir einiges über die Lösungen der homogenen Gleichung

$$
\mathfrak{L F}(z)=l_{0} F(z)+l_{1} F^{\prime}(z)+\cdots+l_{\nu} F^{(v)}(z)+\cdots=0
$$

wissen. Der selbe Ansatz, der bei den analogen Differentialgleichungen endlicher Ordnung zur Auffindung der Lösungen führt, verschafft uns auch einen Teil der Lösungen von (1.5). Wir finden nämlich:

Wenn $\lambda$ eine $m$-fache Nullstelle der Potenzreihe $L(z)$ im Innern ihres Konvergenzkreises ist, und $P(z)$ ein beliebiges Polynom vom Grad $m-1$. bedeutet, so ist

$$
F(z)=P(z) e^{\lambda z}
$$

eine Lösung (Fundamentallösung) der homogenen Gleichung (1.5). Jede endliche lineare Kombination solcher Fundamentallösungen ist wieder eine Lösung von (1.5).

Unter der Voraussetzung, daß Satz III richtig ist, können wir nun den Zusatz $A, B$ beweisen. Nehmen wir an, die Gleichung (1) und damit auch die zugehörige homogene Gleichung (1.5) besitzen nur endlich viele linear unabhängige Lösungen. Dies ist offenbar nur dann möglich, wenn $L(z)$ bloß an endlich vielen Stellen verschwindet. Nun ist nach Voraussetzung $L(z)$ eine ganze Funktion, deren Ordnung 1 nicht übersteigt. Sie muß daher von der Form

$$
L(z)=\left(p_{0}+p_{1} z+\cdots+p_{k} z^{k}\right) e^{c z}, \quad|c| \geqq 0,
$$

sein. Die Operation $\mathfrak{B}$, die durch $L(z)$ erzeugt wird, sieht dann so aus:

$$
\mathfrak{Q} F(z)=p_{0} F(z+c)+p_{1} F^{\prime}(z+c)+\cdots+p_{k} F^{(k)}(z+c) .
$$

Man bestätigt dies, indem man die rechte Seite von (1.6) nach Potenzen von $z$, die rechte Seite von (1.7) nach Potenzen von $c$ entwickelt. Damit ist gezeigt, daß unter der genannten Annahme $F(z)$ mit der linken Seite einer Differentialgleichung endlicher Ordnung gleichwertig ist. 
II.

\section{Beweis des Existenzsatzes in den Fällen A, B, C}

8. Wir wollen die Fälle A, B, C des Satzes III durch einen gemeinsamen Ansatz beweisen, welcher auf Hurwitz [5] zurückgeht.

Wenn $\zeta$ einen Parameter bezeichnet, so gilt

$$
\mathfrak{L} e^{2 \zeta}=l_{0} e^{z \zeta}+l_{1} \zeta e^{z \zeta}+\cdots+l_{\nu} \zeta^{\nu} e^{z \zeta}+\cdots=L(\zeta) e^{z \zeta},
$$

und zwar gleichmäßig in jedem abgeschlossenen Bereich, der nur innere Punkte des Konvergenzkreises von $L(\zeta)$ enthält, also in jedem beschränkten Bereich, wenn einer der Fälle A, B, C vorliegt.

Nun sei $\mathfrak{f}_{n}$ eine im positiven Sinn durchlaufene feste Kreislinie vom Mittelpunkt $\zeta=0$, die im Innern des Konvergenzkreises von $L(\zeta)$ liegt und durch keine Nullstelle von $L(\zeta)$ hindurchgeht. Wir überżeugen uns zunächst, daß

$$
\psi_{n}(z)=\frac{n !}{2 \pi i} \oint_{t_{n}} \frac{e^{z \zeta} d \zeta}{L(\zeta) \zeta^{n+1}} \quad(n=0,1,2, \ldots)
$$

eine Lösung der Gleichung

$$
\mathfrak{L} \psi_{n}(z)=z^{n}
$$

darstellt. Wegen der gleichmäßigen Konvergenz der Reihe in (2.1) sind nämlich die folgenden Umformungen erlaubt:

$$
\begin{aligned}
\mathfrak{L} \psi_{n}(z) & =\frac{n !}{2 \pi i} \oint_{t_{n}} \frac{\mathfrak{L} e^{z^{\zeta}} d \zeta}{L(\zeta) \zeta^{n+1}} \\
& =\frac{n !}{2 \pi i} \oint_{t_{n}} \frac{e^{z \zeta} d \zeta}{\zeta^{n+1}}=z^{n} .
\end{aligned}
$$

Wenn man in (2.2) die Residuen auswertet, so erkennt man, daß $\psi_{n}(z)$ eine ganze Funktion von $z$ ist, und zwar entweder ein Polynom oder eine ganze Funktion vom Mitteltypus der Ordnung 1.

Die rechte Seite von (1) sei durch die Potenzreihe

$$
G(z)=a_{0}+a_{1} z+\cdots+a_{n} z^{n}+\cdots
$$

gegeben ${ }^{4}$ ). Die Beziehung (2.3) legt uns nahe, die Lösung von (1) in der Form

$$
F(z)=a_{0} \psi_{0}(z)+a_{1} \psi_{1}(z)+\cdots+a_{n} \psi_{n}(z)+\cdots
$$

4) Im Fall $A$ kann nämlich als Mittelpunkt des erwähnten Kreises $\Re$ ohne Beschränkung der Allgemeinheit die Stelle $z=0$ angenommen werden. 
anzusetzen. Dieser Ansatz enthält noch unendlich viele verfügbare Parameter, nämlich die Radien der Kreislinien $\mathfrak{f}_{0}, \mathfrak{f}_{1}, \ldots, \mathfrak{f}_{n} \ldots$ Durch passende Wahl dieser Parameter wollen wir eine Funktion $F(z)$ herstellen, die der betreffenden Aussage (Fall A, B, C) des Satzes III voll genügt.

Die Radien der $\mathfrak{f}_{n}$ müssen vor allem so gewählt werden, daß die Reihe $(2.5)$ konvergiert. Ferner soll ihre Summe $F(z)$ das geforderte Tempo des Anwachsens zeigen und eine Lösung von (1) darstellen. Die Funktion $F(z)$ besitzt diese letztere Eigenschaft, wenn die folgende formale Rechnung gerechtfertigt werden kann:

$$
\begin{aligned}
\mathfrak{L F}(z) & =\sum_{\nu=0}^{\infty} l_{\nu} F^{(\nu)}(z)=\sum_{\nu=0}^{\infty} l_{\nu}\left(\sum_{n=0}^{\infty} a_{n} \psi_{n}(z)\right)^{(\nu)} \\
& =\sum_{\nu=0}^{\infty} l_{\nu} \sum_{n=0}^{\infty} a_{n} \psi_{n}^{(\nu)}(z)=\sum_{n=0}^{\infty} a_{n} \sum_{\nu=0}^{\infty} l_{\nu} \psi_{n}^{(\nu)}(z) \\
& =\sum_{n=0}^{\infty} a_{n} \mathfrak{Q} \psi_{n}(z)=\sum_{n=0}^{\infty} a_{n} z^{n}=G(z) .
\end{aligned}
$$

Wir werden zeigen können, daß die Doppelreihe

$$
\sum_{\nu=0}^{\infty} \sum_{n=0}^{\infty} l_{\nu} a_{n} \psi_{n}^{(\nu)}(z)
$$

absolut konvergiert; daher ist die Vertauschung der Summation nach $n$ und $v$ erlaubt. Im Beweis wird enthalten sein, daß die Reihe (2.5) gleichmäßig konvergiert; sie darf daher gliedweise differenziert werden und stellt, wie obige Umformung zeigt, eine Lösung von (1) dar.

9. Die Wahl der Kreise $\mathfrak{f}_{n}$ richtet sich nach dem Verhalten der Potenzreihe $G(z)$ und der erzeugenden Funktion $L(z)$. Um zunächst das Verhalten von $G(z)$ berücksichtigen zu können, führen wir eine regelmäßige Majorante ein. Wir verstehen darunter eine Potenzreihe von der Gestalt

$$
\sum_{n=1}^{\infty}\left(\frac{z}{\varrho_{n}}\right)^{n}
$$

wobei die positiven Zahlen $\varrho_{n}$ so zu bestimmen sind, daß für alle $n=1,2,3, \ldots$ die Ungleichung

und für genügend große $n$

$$
\left|a_{n}\right| \leqq \frac{1}{\varrho_{n}^{n}}
$$

$$
\varrho_{n} \leqq \varrho_{n+1}
$$

gilt. In den einzelnen Fällen werden die $\varrho_{n}$ folgendermaßen gewählt: 
A. Der positive, endliche Konvergenzradius der Potenzreihe (2.4) werde mit $\varrho$ bezeichnet. Wir setzen ${ }^{5}$ )

$$
\frac{1}{\varrho_{n}}=\overline{\operatorname{Max}}\left(\left|a_{n}\right|^{\frac{1}{n}},\left|a_{n+1}\right|^{\frac{1}{n+1}}, \ldots,\left|a_{n+p}\right|^{\frac{1}{n+p}}, \ldots\right) .
$$

Bei dieser Wahl sind die Ungleichungen (2.7) und (2.8) offenbar erfüllt. Ferner gilt

$$
\lim _{n \rightarrow \infty} \frac{1}{\varrho_{n}}=\varlimsup_{n \rightarrow \infty}\left|a_{n}\right|^{\frac{1}{n}}=\frac{1}{\varrho} .
$$

B. Wir definieren $\varrho_{n}$ durch die selbe Formel (2.9) wie im Fall A. Da aber jetzt $G(z)$ eine ganze Funktion ist, gilt an Stelle von (2.10)

$$
\lim _{n \rightarrow \infty} \frac{1}{\varrho_{n}}=\lim _{n \rightarrow \infty}\left|a_{n}\right|^{\frac{1}{n}}=0 \text {. }
$$

C. Im Fall $\mathrm{C}$ soll $\varrho$ die Ordnung der ganzen Funktion $G(z)$ bedeuten; $(\varrho \geqq 1)$. Wir definieren $\varrho_{n}$ durch

$$
-\frac{\log \varrho_{n}}{\log n}=\overline{\operatorname{Max}}\left(\frac{\log \left|a_{n}\right|}{n \log n}, \frac{\log \left|a_{n+1}\right|}{(n+1) \log (n+1)}, \ldots, \frac{\log \left|a_{n+p}\right|}{(n+p) \log (n+p)}, \ldots\right) .
$$

Daraus folgt sofort (2.7), ferner

$$
-\frac{\log \varrho_{n+1}}{\log (n+1)} \leqq-\frac{\log \varrho_{n}}{\log n} \text {. }
$$

(Daraus folgt für $\varrho_{n} \geqq 1$ Ungleichung (2.8), welche übrigens später nicht benützt wird.) Schließlich gilt noch

$$
\lim _{n \rightarrow \infty} \frac{\log \varrho_{n}}{\log n}=-\varlimsup_{n \rightarrow \infty} \frac{\log \left|a_{n}\right|}{n \log n}=\frac{1}{\varrho} .
$$

Man wird bei der Wahl der Kreise $\mathfrak{f}_{n}$ nicht nur die Nullstellen von $L(\zeta)$ zu vermeiden haben, sondern auch die Stellen, wo $L(\zeta)$ klein ist. Dies wird uns durch den folgenden, von Valiron stammenden Hilfssatz ermöglicht [11, S. 89]:

Hilfssatz 1: Es sei $L(\zeta)$ eine ganze Funktion endlicher Ordnung und $k$ eine beliebige Zahl größer als 1. Dann läßt sich eine zugehörige Zahl $H(k)$ finden, mit der Eigenschaft, daß jeder Ring

$$
r_{0}<r \leqq|\zeta| \leqq k \cdot r
$$

mindestens einen Kreis $|\zeta|=r^{\prime}$ enthält, auf welchem

5) Mit $\overline{\operatorname{Max}}\left(\alpha_{1}, \alpha_{2}, \alpha_{3}, \ldots\right)$ werde die obere Weierstraßsche Grenze der Zahlenfolge $\alpha_{1}, \alpha_{2}, \alpha_{3}, \ldots$ bezeichnet; diese obere Grenze braucht nicht erreicht zu sein. 


$$
|L(\zeta)|>[M(k \cdot r)]^{-H(k)}
$$

gilt. Dabei bedeutet $r_{0}$ eine nur von $L(\zeta)$ abhängige $Z$ ahl und $M(r)$ das Maximum von $L(\zeta)$ auf $|\zeta|=r$.

Wir dürfen annehmen, daß $H(k)$ eine im Intervall $1<k \leqq 2$ stetige und monoton fallende Funktion von $k$ ist, welche bei Annäherung an $k=1$ unbeschränkt wächst. Dies läßt sich nämlich durch eine passende Vergrößerung der Werte von $H(k)$ erreichen; die behauptete Ungleichung bleibt dabei bestehen.

10. Nach diesen Vorbereitungen können wir nun zur Bestimmung der Kreislinien $\mathfrak{f}_{0}, \mathfrak{f}_{1}, \mathfrak{F}_{2}, \ldots$ schreiten. Wir benützen dazu eine in den einzelnen Fällen verschieden definierte Folge positiver Zahlen $k_{0}, k_{1}, k_{2}, \ldots$ mit der Eigenschaft

$$
1<k_{n} \leqq 2 \quad(n=0,1,2, \ldots) .
$$

Wir dürfen annehmen, daß das in Hilfssatz 1 vorkommende $r_{0}$ positiv ist. Für $n \geqq 1$ setzen wir

$$
r_{n}=\operatorname{Max}\left(r_{0}, \frac{n}{\varrho_{n}}\right)=n \operatorname{Max}\left(\frac{r_{0}}{n}, \frac{1}{\varrho_{n}}\right) .
$$

Nun wird die Kreislinie $\mathfrak{f}_{n}(n \geqq 0)$ so bestimmt, daß sie konzentrisch im Kreisring

$$
r_{n} \leqq|\zeta| \leqq k_{n} r_{n}
$$

liegt, und daß auf ihr ausnahmslos

$$
\frac{1}{|L(\zeta)|}<\left[M\left(k_{n} r_{n}\right)\right]^{B\left(k_{n}\right)}
$$

gilt. Dies ist nach Hilfssatz 1 wegen (2.16) möglich.

Zur Vorbereitung des Konvergenzbeweises setzen wir

$$
\left|l_{0}\right|+\left|l_{1}\right| z+\left|l_{2}\right| z^{2}+\cdots+\left|l_{\nu}\right| z^{\nu}+\cdots=L^{*}(z) .
$$

Wenn $M(r)$ wie in Hilfssatz 1 definiert wird, so gilt

$$
M(r) \leqq L^{*}(r)
$$

und man hat wegen (2.18) und (2.15) für alle $\zeta$ auf $\mathfrak{\mathfrak { F }}_{n}$

$$
\frac{1}{|L(\zeta)|}<\left[L^{*}\left(2 r_{n}\right)\right]^{H\left(k_{n}\right)} \text {. }
$$

Weiter erhält man unter Berücksichtigung von (2.7), der Fußnote ${ }^{3}$ ) auf 
S. 8, von (2.17), (2.15), (2.16) und (2.20) die für $n \geqq 1$ gültige Abschätzung ${ }^{6}$ )

$$
\begin{aligned}
\left|a_{n} \psi_{n}^{(\nu)}(z)\right| & =\left|\frac{a_{n} n !}{2 \pi i} \oint_{\xi_{n}} \frac{\zeta^{\nu} e^{z \zeta} d \zeta}{\zeta^{n} L(\zeta) \zeta}\right| \\
& \leqq \frac{1}{\varrho_{n}^{n}}(n+2)\left(\frac{n}{e}\right)^{n} \frac{\left(2 r_{n}\right)^{\nu} \exp \left(k_{n} r_{n}|z|\right)}{\left(\frac{n}{\varrho_{n}}\right)^{n}}\left[L^{*}\left(2 r_{n}\right)\right]^{H\left(k_{n}\right)} \\
& =(n+2) \exp \left\{-n+k_{n} r_{n}|z|\right\}\left[L^{*}\left(2 r_{n}\right)\right]^{H\left(k_{n}\right)}\left(2 r_{n}\right)^{\nu} .
\end{aligned}
$$

Folglich gilt nach (2.5)

$$
|F(z)| \leqq\left|a_{0} \psi_{0}(z)\right|+\sum_{n=1}^{\infty}(n+2) \exp \left\{-n+k_{n} r_{n}|z|\right\} \quad\left[L^{*}\left(2 r_{n}\right)\right]^{H\left(k_{n}\right)}
$$

ferner mit Rücksicht auf (2.19)

$$
\begin{aligned}
& \sum_{n=1}^{\infty} \sum_{\nu=0}^{\infty}\left|l_{\nu} a_{n} \psi_{n}^{(\nu)}(z)\right| \\
& \quad \leqq \sum_{n=1}^{\infty}(n+2) \exp \left\{-n+k_{n} r_{n}|z|\right\}\left[L^{*}\left(2 r_{n}\right)\right]^{H\left(k_{n}\right)} \sum_{\nu=0}^{\infty}\left|l_{\nu}\right|\left(2 r_{n}\right)^{\nu} \\
& \quad=\sum_{n=1}^{\infty}(n+2) \exp \left\{-n+k_{n} r_{n}|z|\right\}\left[L^{*}\left(2 r_{n}\right)\right]^{H\left(k_{n}\right)+1}
\end{aligned}
$$

Da $\psi_{0}(z)$ vom Exponentialtypus ist, so geht aus dem Beweis von Satz I hervor, daß

$$
\left|a_{0}\right| \sum_{\nu=0}^{\infty}\left|l_{\nu} \psi_{0}^{(\nu)}(z)\right|
$$

in jedem beschränkten Bereich gleichmäßig konvergiert. Um die Konvergenz der Doppelreihe (2.6) zu beweisen, genügt es daher zu zeigen, daß die rechte Seite von (2.23) beschränkt ist. Dazu müssen die drei Fälle getrennt behandelt werden. Im Fall $\mathrm{C}$ ist dann noch die Größenordnung von (2.22) zu untersuchen.

11. Im Fall $A$ folgt aus (2.10), (2.16), daß für $n \geqq N_{1}$

und daher

$$
\begin{aligned}
& \varrho_{n}>\frac{\varrho}{2} \\
& r_{n}=\frac{n}{\varrho_{n}}
\end{aligned}
$$

$$
L^{*}\left(2 r_{n}\right)<L^{*}\left(\frac{4 n}{\varrho}\right)
$$

6) $\exp (z)=e^{z}$. 
gilt. Da $L^{*}(z)$ für positive $z$ nie verschwindet, können wir, sobald $n \geqq N_{2}$ ist,

$$
L^{*}\left(\frac{4}{\varrho} n\right)=e^{n \cdot \varepsilon_{n}^{2}}, \quad \varepsilon_{n}>0
$$

setzen. Für die $\varepsilon_{n}$, die aus dieser Gleichung bestimmt werden, gilt

$$
\lim _{n \rightarrow \infty} \varepsilon_{n}=0
$$

sonst wäre nämlich das Anwachsen von $L^{*}(z)$ und damit auch das von $L(z)$ stärker als vom Minimaltypus der Ordnung 1, was der Voraussetzung (a) widersprechen würde.

Aus der Bemerkung, die wir im Anschluß an Hilfssatz 1 gemacht haben, geht hervor, daß $H(k)$ im Intervall $1<k \leqq 2$ jeden Wert, der größer als $H(2)$ ist, genau einmal annimmt. Wir können daher wegen (2.27) von einem gewissen $n$ an, d. h. für $n \geqq N_{3} \geqq N_{2}$

$$
H\left(k_{n}\right)=\frac{1}{\varepsilon_{n}}-1
$$

setzen; für kleinere $n$ sei $k_{n}=2$. Die $k_{n}$ sind dadurch eindeutig bestimmt. Die Funktion $H(k)$ ist ferner so beschaffen, daß aus (2.27)

folgt.

$$
\lim _{n \rightarrow \infty} k_{n}=1
$$

Auf Grund von (2.24), (2.25), (2.26) und (2.28) wird die letzte Reihe in (2.23) bis auf endlich viele Anfangsglieder durch

$$
\sum_{n=N}^{\infty}(n+2) \exp \left\{-n+k_{n} \frac{n}{\varrho_{n}}|z|+n \cdot \varepsilon_{n}\right\},
$$

mit $N=\operatorname{Max}\left(N_{1}, N_{3}\right)$ majorisiert. Ist nun $z$ im Kreis $|z|<\varrho$ beliebig aber fest gegeben, so erkennt man unter Benützung von (2.29), (2.10) und (2.27), daß die Glieder der Reihe (2.30) von einem gewissen $n$ an kleiner als $(n+2) \exp (-\alpha n)$ sind, mit $\alpha>0$; d. h. die Reihe konvergiert. Die Konvergenz erfolgt gleichmäßig in jedem abgeschlossenen Teilbereich des Kreises $|z|<\varrho$. Da in einem solchen Bereich die Summe endlich vieler Anfangsglieder der letzten Reihe in (2.23) beschränkt ist, so folgt daraus die gleichmäßige Konvergenz der Reihen in (2.23), ferner die absolute und gleichmäßige Konvergenz der Doppelreihe (2.6) im selben Bereich (man beachte die Bemerkung über $\psi_{0}(z)$ ). Damit haben wir, wie schon früher gezeigt wurde, bewiesen, daß die Reihe (2.5) in jedem abgeschlossenen Teilbereich des Kreises $|z|<\varrho$ gleichmäßig konvergiert and eine in $|z|<\varrho$ reguläre Funktion darstellt, welche (1) befriedigt. In 
der Existenz einer solchen Funktion besteht aber die Behauptung von Satz III im Fall A.

12. Im Fall $B$ wählen wir

$$
k_{n}=2 \quad \text { für } n=0,1,2, \ldots .
$$

Dann hat $H(k)$ einen festen, von $n$ unabhängigen Wert, den wir mit

$$
H\left(k_{n}\right)=H(2)=H
$$

bezeichnen. Im Fall B ist ferner $L(z)$ und damit auch $L^{*}(z)$ (vgl. (2.19)) eine ganze Funktion vom Exponentialtypus; es existieren daher zwei feste Zahlen $S$ und $T$, so daß für jedes positive $r$

$$
L^{*}(r)<S e^{T r}
$$

gilt. Wegen (2.31), (2.32), (2.33) wird die Reihe in der letzten Zeile von (2.23) majorisiert durch

$$
\sum_{n=1}^{\infty} S(n+2) \exp \left\{-n+2 r_{n}[|z|+T(H+1)]\right\} .
$$

Diese Reihe konvergiert aber wegen (2.16), (2.11) in jedem beschränkten Bereich gleichmäßig in bezug auf $z$. Somit ist die Doppelreihe (2.6) absolut und gleichmäßig konvergent; $F(z)$ (vgl. (2.5)) ist daher eine ganze Funktion, die (1) genügt, wie es im Falle $\mathrm{B}$ zu beweisen war.

13. Im Fall $C$ können wir auch $k_{n}=2$ und $H\left(k_{n}\right)=H$, wie in (2.31) und (2.32), setzen. Nun sei $\sigma$ eine Zahl, die beliebig nahe bei $\varrho$, der Ordnung von $G(z)$ liegen darf, aber so, $\mathrm{da} \beta$

$$
\sigma>\varrho \geqq 1
$$

gilt. Die Ordnung von $L(z)$ ist nach Voraussetzung endlich und, falls $\varrho>1$, kleiner als $\varrho^{\prime}$, wo $\varrho^{\prime}$ durch

$$
\frac{1}{\varrho}+\frac{1}{\varrho^{\prime}}=1
$$

definiert ist. Infolgedessen erfüllt die Zahl $\sigma^{\prime}$, welche aus

$$
\frac{1}{\sigma}+\frac{1}{\sigma^{\prime}}=1
$$

bestimmt wird, die Bedingung

$$
\begin{array}{lll}
1<\sigma^{\prime}<\varrho^{\prime} & \text { für } & \varrho>1 \\
1<\sigma^{\prime}<\infty & \text { für } & \varrho=1 .
\end{array}
$$


Sofern $\sigma$ genügend nahe bei $\varrho$ gewählt wurde, ist die Ordnung von $L(z)$ und damit auch die von $L^{*}(z)$ kleiner als $\sigma^{\prime}$; d. h. es gibt eine positive Zahl $\delta$ derart, daß für $r \geqq R$

$$
L^{*}(r)<\exp \left(r^{\sigma^{\prime}(1-\delta)}\right)
$$

gilt. Aus (2.13), (2.14) und (2.34) folgt für $n \geqq N_{1}$

$$
\begin{aligned}
\frac{\log \varrho_{n}-\log 2}{\log n} & >\frac{1}{\sigma}, \\
\frac{\varrho_{n}}{2} & >n^{\frac{1}{\sigma}} \\
\frac{2 n}{\varrho_{n}} & <n^{1-\frac{1}{\sigma}}=n^{\frac{1}{\sigma^{\prime}}} .
\end{aligned}
$$

Schließlich erhalten wir wegen (2.16), (2.31)

$$
k_{n} r_{n}=2 r_{n}<n^{\frac{1}{\sigma^{\prime}}} \quad \text { für } n \geqq \operatorname{Max}\left(N_{1},\left(2 r_{0}\right)^{\sigma^{\prime}}\right) \text {. }
$$

Das ergibt mit (2.37), (2.35)

$$
\begin{aligned}
L^{*}\left(2 r_{n}\right)<L^{*}\left(n^{\frac{1}{\sigma^{\prime}}}\right)< & \exp \left(n^{1-\delta}\right) \\
& \text { für } n \geqq N_{2}=\operatorname{Max}\left(N_{1},\left(2 r_{0}\right)^{\sigma^{\prime}}, R^{\sigma^{\prime}}\right)
\end{aligned}
$$

Die Reihe in der dritten Zeile von (2.23) besitzt wegen (2.38), (2.39) von einem bestimmten Glied an die Majorante

$$
\sum_{n=N_{2}}^{\infty}(n+2) \exp \left\{-n+|z| n^{\frac{1}{\sigma^{\prime}}}+(H+1) n^{1-\delta}\right\},
$$

welche wegen (2.36) in jedem beschränkten Bereich gleichmäßig konvergiert. Da in einem solchen Bereich die hier nicht abgeschätzten Anfangsglieder beschränkt sind, so folgt daraus, daß die Reihe (2.5) in jedem Punkt $z$ konvergiert und eine ganze Funktion darstellt, welche Gleichung (1) befriedigt.

Es bleibt noch die Ordnung von $F(z)$ zu berechnen. Die Zahl $N \geqq N_{2}$ sei so groß gewählt, daß für $n \geqq N$

gilt. Ist dazu noch

$$
-n+H n^{1-\delta}<-\frac{n}{2}
$$

so hat man wegen $(2.35)$

$$
|4 z|^{\sigma}<n,
$$

$$
-n+|z| n^{\frac{1}{\sigma^{\prime}}}+H n^{1-\delta}<-\frac{n}{4} .
$$


Nun wenden wir (2.22) an. Bezeichnen $S$ und $T$ zwei genügend große feste Zahlen, so sind $\left|\psi_{0}(z)\right|$ und die $N$ ersten Glieder der Reihe auf der rechten Seite für alle $z$ kleiner als $S \exp (T|z|)$; der Rest der Reihe besitzt im wesentlichen die selbe Majorante wie (2.23); von einem gewissen $n$ an gilt noch (2.41). Man erhält unter Berücksichtigung von (2.35) für genügend große $|z|$

$$
\begin{aligned}
|F(z)| \leqq & \sum_{n=0}^{N-1} S e^{T|z|} \\
& +\sum_{N \leqq n \leqq|4 z|^{\sigma}}(n+2) \exp \left\{-n+|z| n^{\frac{1}{\sigma^{\prime}}}+H n^{1-\delta}\right\} \\
& \quad+\sum_{n>|4 z|^{\sigma}}(n+2) e^{-\frac{n}{4}} \\
\leqq & N S e^{T|z|}+\left(|4 z|^{\sigma}+2\right)^{2} \cdot \exp \left\{|4 z|^{\sigma}\left(\frac{1}{4}+H\right)\right\}+\sum_{n=1}^{\infty}(n+2) e^{-\frac{n}{4}} .
\end{aligned}
$$

Da $\sigma>1$ ist, so folgt daraus, daß $F(z)$ höchstens die Ordnung $\sigma$ besitzt. Nun durfte aber $\sigma$ beliebig nahe an $\varrho$ gewählt werden. Somit ist die Ordnung von $F(z)$ höchstens gleich $\varrho$; da aber $\mathfrak{L} F(z)=G(z)$ gilt, so kann sie nach Satz II auch nicht kleiner als $\varrho$ sein; d. h. $F(z)$ besitzt genau die Ordnung $\varrho$, was zu beweisen war.

14. Bevor wir zum dritten Kapitel übergehen, welches dem Fall D gewidmet ist, wollen wir den Grund kennenlernen, der die Sonderbehandlung dieses Falles rechtfertigt. Wenn man nacheinander die Voraussetzungen der Sätze in den Fällen A, B, C, D betrachtet, so bemerkt man, daß die Vorschriften über $L(z)$ immer lockerer werden, bis beim Fall D das Minimum dessen erreicht wird, was man von $L(z)$ schließlich doch verlangen darf, daß nämlich ihre Potenzreihe überhaupt ein Konvergenzgebiet besitzt. Im entgegengesetzten Sinn bewegen sich die Vorschriften über $F(z)$ und $G(z)$; sie beginnen mit dem für analytische Funktionen zulässigen Minimum, werden immer schärfer, um schließlich mit einer Schranke für den Typus der Ordnung 1 zu enden. Eine solche Abstufung der Größenordnung muß sich auch bei den Zahlenfolgen zeigen, die wir im Beweis verwendet haben. Wenn man vom Fall $\mathrm{A}$ über $\mathrm{B}$ nach $\mathrm{C}$ übergeht, so wird die Größenordnung der $a_{n}$ und $r_{n}$ kleiner (die der $\varrho_{n}$ größer). Während sich also im Fall A die Kreise $\mathfrak{F}_{n}$,,stürmisch" vergrößern, so wachsen sie im Fall $\mathrm{C}$ nur noch ,,zaghaft" ", und, wenn man die Methode auf den Fall D übertragen würde, könnte man sehen, daß sie sich nur bis zu einer bestimmten endlichen Größe ausweiten. Man hat dann 
aber nicht unendlich viele verschiedene $\mathfrak{f}_{n}$ nötig, sondern es genügt an einem; d. h. wir werden die $\psi_{n}(z)$ (vgl. (2.2)) für alle $n$ als ein Integral über ein und denselben Kreis $\mathfrak{f}$ definieren. Der Konvergenzbeweis für die Reihe (2.5) wird dadurch viel einfacher; jetzt kann man nämlich das Integralzeichen vor die ganze Reihe setzen und gewinnt dadurch gerade die Form, in welcher wir die Lösung im dritten Kapitel aufstellen werden (setzen wir nämlich (2.2) in (2.5) ein und lassen alle $\mathfrak{f}_{n}$ mit $\mathfrak{w}_{0}$ zusammenfallen, so erhalten wir gerade (3.14)). Eine Anpassung des Integrationsweges an das Wachstum von $G(z)$ in den verschiedenen Richtungen führt dann zum Zusatz D 1 zu Satz III.

Zusammenfassend läßt sich sagen: Die im II. Kapitel angewandte Methode ist auch im Fall D brauchbar, führt aber sofort zu einer einfacheren Darstellung der Lösung. Diese kann aber auch auf einem ganz andern Weg gefunden werden, wie das III. Kapitel zeigen wird.

III.

\section{Ganze Funktionen vom Exponentialtypus}

15. Ich beginne mit der Erläuterung einiger Definitionen und Tatsachen, die ganze Funktionen vom Exponentialtypus betreffen; man vergleiche dazu [9, S. 571-585]. Wir werden dabei von folgender Vereinfachung der Bezeichnung Gebrauch machen: Statt ,Das Anwachsen der Funktion $F(z)$ ist höchstens vom Typus $t$ der Ordnung $1^{\text {" schreiben }}$ wir nur ,Die Funktion $F(z)$ ist höchstens vom Typus $t^{\circ}$, und statt ,Das Anwachsen der Funktion $F(z)$ ist höchstens vom Minimaltypus der Ordnung $1^{\prime \prime}$ schreiben wir "Die Funktion $F(z)$ ist höchstens vom Typus 0 ". Alle Aussagen, in denen $t$ vorkommt, gelten sinngemäß auch für $t=\mathbf{0}$.

Die Potenzreihe $\quad F(z)=c_{0}+c_{1} z+c_{2} z^{2}+\cdots+c_{n} z^{n}+\cdots$

sei höchstens vom Typus $t$, oder, was damit gleichbedeutend ist, es gelte

$$
\varlimsup_{n \rightarrow \infty}\left|n ! c_{n}\right|^{\frac{1}{n}} \leqq t .
$$

Der Reihe (3.1) läßt sich die Potenzreihe

$$
f(\zeta)=\frac{c_{0}}{\zeta}+\frac{1 ! c_{1}}{\zeta^{2}}+\frac{2 ! c_{2}}{\zeta^{3}}+\cdots+\frac{n ! c_{n}}{\zeta^{n+1}}+\cdots
$$

zuordnen; ihre Summe $f(\zeta)$ wird die Borelsche Transformierte von $F(z)$ genannt. Aus (3.2) folgt, daß die Reihe (3.3) im Äußern des Kreises $|\zeta|=t$ konvergiert und dort eine reguläre Funktion darstellt. 
Zur genaueren Erfassung des Wachstums von $F^{\prime}(z)$ dient die reellwertige Funktion $h(\varphi)$, welche folgendermaßen definiert ist:

$$
h(\varphi)=\varlimsup_{r \rightarrow \infty} \frac{\log \left|F\left(r e^{i \varphi}\right)\right|}{r} .
$$

Man nennt $h(\varphi)$ den Indikator von $F(z)$. Der Indikator mißt also das Anwachsen von $F(z)$ längs eines Halbstrahles, der mit der positiven reellen Achse den Winkel $\varphi$ einschließt. Die Funktion $h(\varphi)$ kann als Stützfunktion eines ganz im Endlichen gelegenen abgeschlossenen und konvexen Bereiches aufgefaßt werden. Dieser Bereich wird das Indikator diagramm von $F(z)$ genannt und mit $\mathfrak{I}$ bezeichnet. Genauer gesagt, liegt ein Punkt $z=x+i y$ dann und nur dann in $\mathfrak{J}$, wenn für alle $\varphi$

$$
\Re\left(z e^{-i \varphi}\right)=x \cos \varphi+y \sin \varphi \leqq h(\varphi)
$$

gilt. Ferner ist für alle $\varphi$ die Ungleichung $h(\varphi) \leqq t$ erfüllt; d. h. $\mathfrak{J}$ ist ein echter oder unechter Teilbereich des Kreises $|z| \leqq t$.

Es gibt konvexe Bereiche, in deren Außenraum die durch die Reihe (3.3) definierte Funktion $f(\zeta)$ ausnahmslos regulär ist; ein solcher Bereich ist z. B. der Kreis $|\zeta| \leqq t$. Der Durchschnitt aller dieser Bereiche wird das konjugierte Diagramm genannt und mit $\overline{\mathfrak{J}}$ bezeichnet. Der abgeschlossene und konvexe Bereich $\overline{\mathfrak{J}}$ ist im Kreis $|\zeta| \leqq t$ enthalten und enthält alle Singularitäten von $f(\zeta)$. Es gilt nun der

Hilfssatz 2. [9, S. 585.] Das konjugierte Diagramm $\overline{\mathfrak{J}}$ ist (wie schon die Bezeichnung ausdrückt), das Spiegelbild des Indikatordiagrammes $\mathfrak{I}$ in bezug aut die reelle Achse.

Die Funktion $F(z)$ läßt sich folgendermaßen durch ihre Borelsche Transformierte $f(\zeta)$ ausdrücken:

$$
F(z)=\frac{1}{2 \pi i} \oint_{\mathfrak{w}} e^{z \xi} f(\zeta) d \zeta
$$

Das Integral ist in positivem Sinn über einen geschlossenen Weg w zu erstrecken, der das konjugierte Diagramm $\overline{\mathfrak{I}}$ im Innern enthält. Man verifiziert diese Formel, indem man die Funktionen im Integranden durch ihre Potenzreihen ersetzt und über einen Weg, auf dem (3.3) gleichmäßig konvergiert, gliedweise integriert. Weiter gilt folgender

Hilfssatz 3. (Für den Beweis vergleiche [9, S. 584]). Es sei $\mathfrak{B}$ ein im Endlichen liegender, konvexer und abgeschlossener Bereich der komplexen Ebene, $\mathfrak{w}$ eine geschlossene, doppelpunltlose Kurve, die $\overline{\mathfrak{B}}$, das Spiegelbild 
von $\mathfrak{B}$ in bezug auf die reelle Achse, im Innern enthält und $v(\zeta)$ eine Funktion, die auf $\mathfrak{w}$ und zwischen $\mathfrak{w}$ und $\mathfrak{B}$ eindeutig und regulär ist. Dann stellt das über $\mathfrak{w}$ erstreckte Integral

$$
\frac{1}{2 \pi i} \oint_{\mathfrak{w}} e^{z \zeta} v(\zeta) d \zeta
$$

eine Funktion vom Exponentialtypus dar, deren Indikatordiagramm in $\mathfrak{B}$ enthalten ist.

Die Definitionen und Sätze dieses Abschnittes gelten auch für den Fall, daß $F(z)$ höchstens vom Typus 0 ist. Der Indikator $h(\varphi)$ wird dann identisch Null, das Indikatordiagramm und das konjugierte Diagramm fallen mit dem Nullpunkt zusammen. Dies gilt speziell auch dann, wenn die Ordnung von $F(z)$ kleiner als 1 ist. In diesem Fall besteht aber darüber hinaus noch der

Hilfssatz 4. Es sei $F(z)$ eine Funktion der Ordnung $\varrho$, mit $\varrho<1, f(\zeta)$ ihre Borelsche Transformierte, w ein Weg, der den Nullpunlt einmal umläuft und $v(\zeta)$ eine Funktion, die auf $\mathfrak{w}$ und im Innern von $\mathfrak{w}$ regulär ist oder höchstens im Nullpunkt einen Pol hat. Dann stellt das über $\mathfrak{w}$ erstreckte Integral

$$
\frac{1}{2 \pi i} \oint_{\mathfrak{w}} e^{z \zeta} f(\zeta) v(\zeta) d \zeta
$$

eine ganze Funktion dar, deren Ordnung höchstens gleich @ ist.

Für wo kann ein beliebiger Kreis $|\zeta|=r<\delta$ gewählt werden, wenn $v(\zeta)$ in $0<|\zeta|<\delta$ regulär ist. Nach Voraussetzung gilt für die Koeffizienten der Reihe (3.1)

$$
\varlimsup_{n \rightarrow \infty} \frac{n \log n}{-\log \left|c_{n}\right|}=\varrho .
$$

Wir bemerken, daß $f\left(w^{-1}\right)$ eine ganze Funktion von $w$ ist, deren Ordnung $\varrho^{\prime}$ aus den Koeffizienten der Reihe (3.3) berechnet werden kann. Man erhält mit Berücksichtigung der Fußnote ${ }^{3}$ ) auf S. 158

$$
\varlimsup_{n \rightarrow \infty} \frac{n \log n}{-\log n !\left|c_{n}\right|}=\frac{\varrho}{1-\varrho}=\varrho^{*} .
$$

Bedeutet daher $\sigma$ eine beliebige Zahl größer als $\varrho^{*}$, so besteht, sofern $|w|^{-1}>r_{0}$ ist, die Ungleichung

$$
\left|f\left(w^{-1}\right)\right|<e^{|w|^{-\sigma}} .
$$


Wir setzen nun

$$
r=|z|^{\frac{-1}{1+\sigma}}
$$

und betrachten nur solche $z$, für welche $r<\operatorname{Min}\left(r_{\mathbf{0}}^{-1}, \delta\right)$ wird. Mit $m$ werde die Ordnung des Poles von $v(\zeta)$ im Nullpunkt bezeichnet ( $m=0$, wenn $v(\zeta)$ dort regulär ist). Dann erlaubt das Integral (3.5) folgende Abschätzung:

$$
\begin{aligned}
\left.\frac{1}{2 \pi}\right|_{\mathfrak{w}} \oint^{z \zeta} f(\zeta) v(\zeta) d \zeta \mid & <\exp \left(|z| r+r^{-\sigma}\right) A r^{1-m} \\
& =A|z|^{B} \exp \left(2|z|^{\widetilde{1+\sigma}}\right)<e^{|z|^{e+\varepsilon}},
\end{aligned}
$$

wo $A$ und $B$ feste Zahlen bedeuten. Da $\sigma$ beliebig nahe an $\varrho^{*}$ gewählt werden kann, so erkennt man unter Beachtung der zweiten Gleichung in (3.6), daß für jedes noch so kleine positive $\varepsilon$ eine solche Ungleichung erfüllt ist, sofern nur $|z|$ genügend groß gewählt wird; dies ist aber die Behauptung des Hilfssatzes.

16. Ich werde in diesem Kapitel von einem Satz Gebrauch machen, den mir Prof. Pólya mitteilte; es ist dies

Hilfssatz 5. Es sei w ein geschlossener, doppelpunktloser Weg in der $z$-Ebene, $R(z)$ eine ganze Funktion, in deren Entwicklung

$$
R(z)=b_{0}+b_{1} z+b_{2} z^{2}+\cdots
$$

alle Koeffizienten $b_{0}, b_{1}, b_{2}, \ldots$ von Null verschieden sind, $Q(z)$ eine entlang $\mathfrak{w}$ reguläre und eindeutige Funktion. Dann ist

$$
\oint_{\mathfrak{w}} R(z \zeta) Q(\zeta) d \zeta
$$

dann und nur dann identisch Null in $z$, wenn $Q(z)$ im Innern von $\mathfrak{w}$ regulär ist.

Daß diese Bedingung hinreichend ist, folgt aus dem Cauchyschen Integralsatz; wir haben also nur zu beweisen, da $\beta$ sie auch notwendig ist.

Es gibt zwei geschlossene Kurven $\mathfrak{a}$ und $\mathfrak{b}$, von denen $\mathfrak{a}$ ganz im Äußern, $\mathfrak{b}$ ganz im Innern von $\mathfrak{w}$ liegt, und auf welchen $Q(z)$ auch noch regulär ist. Mit Hilfe der Cauchyschen Integralformel kann man $Q(z)$ nun folgendermaßen schreiben

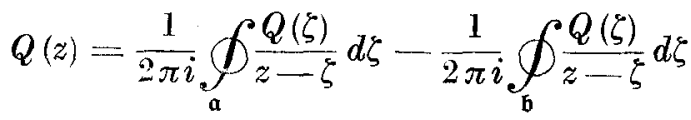

$$
\begin{aligned}
& =Q_{\mathfrak{a}}(z)-Q_{\mathfrak{b}}(z) \text {. }
\end{aligned}
$$


Die Funktion $Q(z)$ läßt sich also als Differenz zweier Funktionen darstellen, von denen $Q_{\mathfrak{a}}(z)$ im Innern von $\mathfrak{a}, Q_{\mathfrak{b}}(z)$ im Äußern von $\mathfrak{b}$ regulär und eindeutig ist. Die Funktion $Q_{\mathfrak{b}}(z)$ besitzt daher eine Reihenentwicklung von der Form

$$
Q_{\mathrm{b}}(z)=\frac{q_{0}}{z}+\frac{q_{1}}{z^{2}}+\frac{q_{2}}{z^{3}}+\cdots,
$$

welche auf jedem Kreis $\mathfrak{f}$, der $\mathfrak{b}$ im Innern enthält, gleichmäßig konvergiert. Indem man den Integrationsweg deformiert und auf dem neuen Weg sowohl $Q_{\mathrm{b}}(\mathrm{z})$ durch ihre Reihe wie auch $R(z)$ durch die Reihe (3.7) ersetzt, erhält man schließlich

$$
\begin{aligned}
& \oint_{\mathfrak{w}} R(z \zeta) Q(\zeta) d \zeta=\oint_{\mathfrak{w}} R(z \zeta)\left(Q_{\mathfrak{a}}(\zeta)-Q_{\mathfrak{b}}(\zeta)\right) d \zeta \\
=- & \oint_{\mathfrak{w}} R(z \zeta) Q_{\mathfrak{b}}(\zeta) d \zeta=-\oint_{\mathfrak{z}} \sum_{\nu=0}^{\infty} \sum_{n=0}^{\infty} b_{\nu} q_{n} z^{\nu} \zeta^{\nu-n-1} d \zeta \\
= & -2 \pi i\left(b_{0} q_{0}+b_{1} q_{1} z+b_{2} q_{2} z^{2}+\cdots\right) .
\end{aligned}
$$

Die Doppelreihe unter dem Integralzeichen ist als Produkt zweier absolut und gleichmäßig konvergenter Potenzreihen selbst gleichmäßig konvergent und kann daher gliedweise integriert werden. Da alle Koeffizienten $b_{0}, b_{1}, b_{2}, \ldots$ von Null verschieden sind, so kann die Reihe in der dritten Zeile nur dann identisch verschwinden, wenn

$$
q_{\mathbf{0}}=q_{1}=q_{2}=\cdots=0
$$

gilt. Also reduziert sich $Q(z)$ auf $Q_{\mathfrak{a}}(z)$, eine im Innern von w reguläre Funktion, wie zu beweisen war.

17. Nun sind wir imstande die Sätze I bis III im Fall D zu beweisen. Zunächst haben wir den Beweis nachzuholen, daß die Bedingung $(d)$ von Satz I auch hinreichend ist. Gleichzeitig damit wird gezeigt, daß die Operation $\mathbb{Q}$ unter den Voraussetzungen dieses Satzes die Darstellung

$$
\mathcal{L} F^{\prime}(z)=\frac{1}{2 \pi i} \oint_{\mathfrak{w}} e^{z \zeta} f(\zeta) L(\zeta) d \zeta
$$

besitzt. Dabei ist im positiven Sinn über einen Weg w zu integrieren, der im Gebiet gleichmäßiger Konvergenz der Reihe (3) verläuft und das konjugierte Diagramm $\widetilde{\mathfrak{J}}$ von $F(z)$ im Innern enthält. Das ist möglich, weil $\overline{\mathfrak{J}}$ im Kreis $|\zeta| \leqq t$ enthalten ist und daher wegen $(d)$ ganz im Innern des Konvergenzkreises von $L(z)$ liegt. Da $f(\zeta)$ außerhalb $\mathfrak{\Im}$ regulär ist, so hängt der Wert des Integrales (3.9) nicht von der speziellen Wahl von 
$\mathfrak{w}$ ab. Wir können daher für $\mathfrak{w}$ den Kreis $|\zeta|=t+\varepsilon>t$ nehmen und $\varepsilon$ so klein wählen, daß darauf nicht nur (3.3) sondern auch noch (3) gleichmäßig konvergiert (vgl. (d), (3.2)). Dann sind folgende Umformungen erlaubt

$$
\begin{gathered}
\frac{1}{2 \pi i} \oint_{\mathbf{w}} e^{z \zeta} f(\zeta) L(\zeta) d \zeta=\frac{1}{2 \pi i} \oint_{\mathfrak{w}} \sum_{k=0}^{\infty} \sum_{n=0}^{\infty} \sum_{\nu=0}^{\infty} \frac{z^{k} \zeta^{k} n ! c_{n} l_{\nu} \zeta^{\nu}}{k ! \zeta^{n+1}} d \zeta \\
\quad=\sum_{\nu=0}^{\infty} l_{\nu} \sum_{n=\nu}^{\infty} \frac{n ! c_{n}}{(n-\nu) !} z^{n-\nu}=\sum_{\nu=0}^{\infty} l_{\nu} F^{(\nu)}(z)=\mathfrak{L} F(z) .
\end{gathered}
$$

Die dreifache Reihe unter dem Integralzeichen ist ja für jedes feste $z$ in bezug auf $\zeta$ gleichmäßig konvergent und gestattet daher die gliedweise Integration. Mit der Konvergenz der Reihen in der zweiten Zeile ist auch die Aussage von Satz I im Fall D bewiesen.

Die Integraldarstellung (3.9) erlaubt uns auch, Satz II im Fall D und zwar gleich in der verschärften Form von Zusatz D 1 zu beweisen. Man hat dazu Hilfssatz 3 anzuwenden, indem man $\mathfrak{B}$ mit dem Indikatordiagramm von $F(z)$ zusammenfallen läßt und $v(\zeta)=f(\zeta) L(\zeta)$ setzt. Auf die gleiche Art erhält man die Aussage von Zusatz D 2, indem man Hilfssatz 4 anwendet und dort $v(\zeta)=L(\zeta)$ setzt.

18. Wir gehen nun zum Existenzsatz III über, indem wir ihn gerade zusammen mit Zusatz D 1 beweisen. Es sollen $\mathfrak{B}$ und $\overline{\mathfrak{B}}$ die dort erklärte Bedeutung haben. Wir wollen uns zunächst folgende Frage stellen: Wie muß eine Funktion $F(z)$ beschaffen sein, die eine Lösung von (1) darstellt und deren Indikatordiagramm in $\mathfrak{B}$ enthalten ist? Eine solche Funktion kann zunächst in der Form (3.4) geschrieben werden. Da nach Hilfssatz 2 das konjugierte Diagramm von $F^{\prime}(z)$ in $\overline{\mathfrak{B}}$ enthalten ist, so kann als Integrationsweg $\mathfrak{w}$ jede im positiven Sinn durchlaufene Kurve genommen werden, die $\bar{B}$ im Innern enthält. Wir wählen $\mathfrak{w}$ so, daß $L(\zeta)$ auf $\mathfrak{w}$ und zwischen $\mathfrak{w}$ und $\overline{\mathfrak{B}}$ regulär und nullstellenfrei ist. Mit diesem $\mathfrak{w}$ kann nun die linke Seite von (1) in der Form (3.9) dargestellt werden. Ferner folgt aus Hilfssatz 2, daß auch das konjugierte Diagramm $\overline{\mathfrak{J}}$ von $G(z)$, der rechten Seite von (1), in $\overline{\mathfrak{B}}$ enthalten ist; deshalb läßt sich auch $G(z)$ in der Form

$$
G(z)=\frac{1}{2 \pi i} \oint_{\mathfrak{w}} e^{z \zeta} g(\zeta) d \zeta
$$

durch ihre Borelsche Transformierte ausdrücken. Damit findet man, daß die Gleichung (1) mit

äquivalent ist.

$$
\oint_{\mathfrak{w}} e^{z \zeta}(f(\zeta) L(\zeta)-g(\zeta)) d \zeta=0
$$


Wann ist nun dieses Integral identisch Null in $z$ ? Diese Frage beantwortet uns Hilfssatz 5; wir haben nur $R(z)=e^{z}$ und

$$
Q(\zeta)=f(\zeta) L(\zeta)-g(\zeta)
$$

zu setzen und finden, daß $Q(\zeta)$ innerhalb w regulär sein muß. Löst man (3.12) nach $f(\zeta)$ auf und setzt in (3.4) ein, so gelangt man zum Resultat, daß eine Lösung von (1), deren Indikatordiagramm in $\mathfrak{B}$ enthalten ist, die Form

$$
F(z)=\frac{1}{2 \pi i} \oint_{\mathfrak{w}} e^{z \xi} \frac{g(\zeta)+Q(\zeta)}{L(\zeta)} d \zeta
$$

besitzen $m u \beta$, wobei $Q(\zeta)$ auf $\mathfrak{w}$ und innerhalb $\mathfrak{w}$ regulär ist.

Nun wollen wir umgekehrt zeigen, daß das Integral (3.13) stets eine Lösung von (1) bildet, deren Indikatordiagramm in $\mathfrak{B}$ enthalten ist. Die letztere Behauptung ergibt sich aus Hilfssatz 3, wenn man dort für $v(\zeta)$ den Bruch im Integranden von (3.13) einsetzt. (Man beachte, wie wir w gewählt haben.) Setzen wir jetzt (3.13) in die linke Seite von (1) ein! Da die Reihe in (2.1) auf $\mathfrak{w}$ gleichmäßig konvergiert, darf das Zeichen $\mathbb{I}$ mit dem Integralzeichen vertauscht werden. Man erhält mit (2.1), (3.10)

$$
\begin{aligned}
& \mathcal{L} F(z)=\frac{1}{2 \pi i} \oiint_{\mathfrak{w}} \mathcal{L} e^{z \xi} \frac{g(\zeta)+Q(\zeta)}{L(\zeta)} d \zeta=\frac{1}{2 \pi i} \oint_{\mathfrak{w}} e^{z \vdots}(g(\zeta)+Q(\zeta)) d \zeta \\
& =\frac{1}{2 \pi i} \oint_{\mathfrak{w}} e^{z \zeta} g(\zeta) d \zeta=G(z) \text {. }
\end{aligned}
$$

$F(z)$ ist also eine Lösung von (1).

Schließlich bleibt noch nachzuweisen, daß die Lösung, die wir in der Form (3.13) gefunden haben, mit der rechten Seite von (4) gleichbedeutend ist. Zunächst soll gezeigt werden, wie man $\operatorname{das} F_{0}(z)$ erhält. Wir wählen einen geschlossenen Weg $\mathfrak{w}_{0}$, der das konjugierte Diagramm $\overline{\mathfrak{J}}$ von $G(z)$ im Innern enthält, es aber so eng umschließt, daß $L(\zeta)$ auf $\mathfrak{w}_{0}$ und zwischen $\mathfrak{m}_{0}$ und $\overline{\mathfrak{I}}$ regulär und nullstellenfrei ist. Dann stellt das im positiven Sinn über $\mathfrak{w}_{0}$ erstreckte Integral

$$
F_{0}(z)=\frac{1}{2 \pi i} \oint_{\mathfrak{w}_{0}} \frac{e^{z \zeta} g(\zeta)}{L(\zeta)} d \zeta
$$

als Spezialfall von (3.13) eine Lösung von (1) dar, deren Indikatordiagramm in $\mathfrak{J}$ enthalten ist. Da aber $\mathfrak{L F}(z)=G(z)$ gilt, so muß umgekehrt auch $\mathfrak{J}$ im Indikatordiagramm von $F_{0}(z)$ enthalten sein; dies folgt ja aus Satz II, Zusatz D 1; die Indikatordiagramme von $F_{0}(z)$ und $G(z)$ müssen 
daher zusammenfallen. Wir haben damit die den Fall D betreffende Aussage von Satz III bewiesen.

Die rechte Seite von (3.13) läßt sich nun folgendermaßen umformen:

$$
\begin{aligned}
F(z)=\frac{1}{2 \pi i} \oint_{\mathfrak{w}_{0}} \frac{e^{z \zeta} g(\zeta)}{L(\zeta)} d \zeta & +\frac{1}{2 \pi i}\left[\oint_{\mathfrak{w}} \frac{e^{z \zeta} g(\zeta)}{L(\zeta)} d \zeta-\oint_{\mathfrak{w}_{0}} \frac{e^{z \zeta} g(\zeta)}{L(\zeta)} d \zeta\right] \\
& +\frac{1}{2 \pi i} \oint_{\mathfrak{w}} \frac{e^{z \zeta} Q(\zeta)}{L(\zeta)} d \zeta \\
=\quad & \quad+P_{m_{1}}(z) e^{\lambda_{1} z}+\cdots+P_{m_{l}}(z) e^{\lambda_{l} z} .
\end{aligned}
$$

Beachtet man, daß $Q(z)$ innerhalb $\mathfrak{w}$ und $g(\zeta)$ zwischen $\mathfrak{w}_{0}$ und $\mathfrak{w}$ regulär sind, und berechnet man die Residuen, so zeigt sich, daß die Symbole in der letzten Zeile die gleiche Bedeutung wie in (4) haben. Damit haben wir auch den Zusatz D 1 zu. Satz III bewiesen.

Schließlich bleibt noch der Beweis für Zusatz D 2 zu leisten. Überträgt man die vorangehenden Überlegungen auf den hier vorliegenden Spezialfall, so reduzieren sich die Bereiche $\mathfrak{J}, \overline{\mathfrak{J}}, \mathfrak{B}, \overline{\mathfrak{B}}$ auf den Nullpunkt. Die Kurve $\mathfrak{w}_{0}$ (die hier auch die Rolle von $\mathfrak{w}$ spielt), muß so eng um den Nullpunkt gelegt werden, daß $L(z)$ auf $\mathfrak{w}_{0}$ und im Innern von $\mathfrak{w}_{0}$ regulär und bis auf $z=0$ nullstellenfrei bleibt. Eine Lösung von (1), deren Ordnung kleiner als 1 ist, muß, nach (3.13), die Form

$$
F(z)=\frac{1}{2 \pi i} \oint_{\mathfrak{w}_{0}} \frac{e^{z \zeta} g(\zeta)}{L(\zeta)} d \zeta+\frac{1}{2 \pi i} \oint_{\mathfrak{w}_{0}} \frac{e^{z \zeta} Q(\zeta)}{L(\zeta)} d \zeta
$$

besitzen, wobei $Q(\zeta)$ auf $\mathfrak{w}_{0}$ und im Innern von $\mathfrak{w}_{0}$ regulär ist. Wendet man auf das erste Integral auf der rechten Seite, das wie in (3.14) mit $F_{0}(z)$ bezeichnet werde, den Hilfssatz 4 an, indem man dort $F(z)$ durch $G(z)$ und $v(\zeta)$ durch $[L(\zeta)]^{-1}$ ersetzt, so findet man, daß $F_{0}(z)$ höchstens von der Ordnung $\varrho$ sein kann. Da aber $\mathfrak{L} F(z)=G(z)$ gilt, so kann nach Zusatz $\mathrm{D} 2$ zu Satz II die Ordnung von $F_{0}(z)$ auch nicht kleiner als $\varrho$ sein; sie ist daher gleich $\varrho$. Das zweite Integral in (3.15) ergibt ein Polynom, das die Eigenschaften von $P_{m}(z)$ in Zusatz D 2 zu Satz III besitzt. Damit haben wir auch diesen Zusatz bewiesen.

19. Ich mache noch einige Bemerkungen zu der Form, in welcher wir die Lösung in den Fällen A, B und C gefunden haben (und in welcher wir sie auch im Fall $\mathrm{D}$ finden könnten).

Wenn man in (2.2) die Residuen auswertet, so sieht man, daß $\psi_{n}(z)$ von folgender Form ist:

$$
\psi_{n}(z)=\varphi_{n}(z)+\pi_{n, r}(z) .
$$


Dabei bedeutet $\varphi_{n}(z)$ ein Polynom, dessen Grad unter der Annahme $L(0) \neq 0$ gleich $n$ ist (sonst erhöht er sich um die Vielfachheit der Nullstelle von $L(z)$ im Nullpunkt). Wenn wir an Stelle von (1) die Differenzengleichung $F(z+1)-F(z)=z^{n}$ vor uns haben, so sind die $\varphi_{n}(z)$ mit den Bernoullischen Polynomen identisch. Weiter bezeichnet $\pi_{n},{ }_{r}(z)$ eine lineare Kombination solcher Fundamentallösungen ${ }^{7}$ ) der homogenen Gleichung, die mit denjenigen Nullstellen von $L(z)$ gebildet sind, die im Kreis $|z|<r$ liegen. Da auch $\mathfrak{Q} \varphi_{n}(z)=z^{n}$ ist, so befriedigt die Reihe

$$
a_{0} \varphi_{0}(z)+a_{1} \varphi_{1}(z)+a_{2} \varphi_{2}(z)+\cdots
$$

„formal“ die Gleichung (1); sie wird aber im allgemeinen divergieren. Man kann sie aber durch Hinzufügung der Summanden $\pi_{n},{ }_{r}(z)$ konvergent machen. Vgl. [5].

Die Form, in welcher wir die Lösung von (1) gewonnen haben, besitzt eine gewisse Ähnlichkeit mit der Form, in welcher die Aufgabe, eine ganze Funktion mit vorgeschriebenen Nullstellen $b_{0}, b_{1}, b_{2}, \ldots$ anzugeben, gelöst wird. Bei dieser Gegenüberstellung entsprechen sich diese Nullstellen und die Koeffizienten $a_{0}, a_{1}, a_{2}, \ldots$ der gegebenen Funktion $G(z)$. Der Ansatz (3.16) für die Lösung von (1) ist analog dem Ansatz

$$
\left(1-\frac{z}{b_{0}}\right)\left(1-\frac{z}{b_{1}}\right)\left(1-\frac{z}{b_{2}}\right) \cdots
$$

für eine Funktion, die in $b_{0}, b_{1}, b_{2}, \ldots$ verschwindet. Beide Entwicklungen lassen sich konvergent machen, indem man zu dem allgemeinen Glied in (3.16) den konvergenzerzeugenden Summanden $a_{n} \psi_{n},{ }_{r}(z)$, bzw. in (3.17) einen geeigneten konvergenzerzeugenden Faktor $\exp \left(P\left(z / b_{n}\right)\right)$ hinzufügt; es wird also im ersten Fall eine Lösung der homogenen Gleichung, im zweiten eine nullstellenfreie Funktion hinzugefügt. Ferner erhalten wir die allgemeine Lösung von (1) durch Addition der allgemeinen Lösung der homogenen Gleichung zu einer speziellen der inhomogenen; ebenso wird eine beliebige ganze Funktion mit den Nullstellen $b_{0}, b_{1}, b_{2}, \ldots$ durch Multiplikation einer speziellen solchen Funktion mit einer beliebigen nullstellenfreien ganzen Funktion erhalten. Bei der Produktdarstellung einer ganzen Funktion der endlichen Ordnung $\varrho$ ist die Ordnung der Exponentialfaktoren durch $\varrho$ begrenzt; ist $G(z)$ in (1) vom Typus $t$ der Ordnung 1 (Fall D), so ist der Typus der konvergenzerzeugenden Summanden durch $t$ begrenzt.

7) Vgl. Nr. 7 . 


\section{Curriculum vitae}

Ich wurde am 16. Januar 1912 in Rüschlikon (Zürich) geboren, besuchte dort während 6 Jahren die Primarschule und trat im Frühling 1924 in das kantonale Gymnasium Zürich ein, um im Herbst 1930 mit der Maturität abzuschließen. Hierauf studierte ich an der Abteilung für Mathematik und Physik der Eidgenössischen Technischen Hochschule und erwarb im Frühjahr 1935 das Diplom als Mathematiker. Anschließend studierte ich noch ein Semester an der Universität Hamburg. Vom Herbst 1935 bis zum Sommer 1936 durfte ich als Assistent für Darstellende Geometrie bei Herrn Prof. Saxer tätig sein. Nachher unterrichtete ich als Vikar und Hilfslehrer am Evangelischen Lehrerseminar Unterstraß und am kantonalen Gymnasium Zürich; im Wintersemester 1937/38 war ich außerdem Assistent für Höhere Mathematik bei Herrn Prof. Pólya.

Ich danke an dieser Stelle Herrn Prof. Pólya für die Anregung zu dieser Arbeit und für die vielen wertvollen Ratschläge während der Ausarbeitung recht herzlich.

Rüschlikon, den 5. Juli 1938.

Hermann Muggli. 